\title{
Optimización de la capacidad de producción en una empresa de alimentos usando simulación de eventos discretos
}

\author{
Optimizing production capacity in a food company using discrete event simulation
}

\author{
Lina Vanessa Peña Ariza ${ }^{1} \quad$ Heriberto Alexander Felizzola Jimenez ${ }^{1 *}$ \\ Recibido 20 de junio de 2018, Aceptado 31 de enero de 2019 \\ Received: June 20, 2018 Accepted: January 31, 2019
}

\begin{abstract}
RESUMEN
El presente trabajo muestra la aplicación de simulación de eventos discretos, con el propósito de evaluar propuestas de mejora en la capacidad del proceso de producción y por ende la eficiencia de una empresa de alimentos, mediante el planteamiento y comparación de escenarios. La metodología usada para su desarrollo se dividió en cinco (5) fases: fase I: caracterización del proceso y cuantificación de los recursos actuales, fase II: análisis de entrada, fase III: desarrollo y validación del modelo usando el software Tecnomatix Plant simulation, fase IV: propuesta y simulación de tres escenarios, fase V: análisis de escenarios, con respecto a los resultados obtenidos en los indicadores de desempeño: Throughput, porcentaje de utilización y porcentaje de tiempo de espera en cada operación. El escenario que generó mejores resultados frente al análisis realizado fue el tercero, que correspondía a la reprogramación de la producción, con el que se logró aumentar el throughput en 38,70\%, 9,57\% y 56,62\% para las tortillas de maíz, tortillas de promasa y tortillas de harina de trigo, respectivamente. Por otro lado, se aumentó el porcentaje de utilización y se redujo el porcentaje de tiempo de espera de cada operación, y con esto se logró aumentar la eficiencia a través del aumento de la capacidad de producción.
\end{abstract}

Palabras clave: Simulación de eventos discretos, procesos productivos, asignación de recursos, throughput, mejora de procesos.

\begin{abstract}
The present work shows the application of simulation of discrete events, to evaluate proposals to improve the capacity of the production process. Therefore, the efficiency of a food company through the approach and comparison of scenarios. The methodology used for its development was divided into five (5) phases, phase I: characterization of the process and quantification of current resources, phase II: input analysis, phase III: development and validation of the model using Tecnomatix Plant simulation software, phase IV: proposal and simulation of three scenarios, phase V: scenario analysis, concerning the results obtained in the performance indicators: Throughput, percentage of use and waiting time in each operation. The scenario that generated better results compared to the analysis was the third, which corresponded to the reprogramming of production, which was able to increase throughput by $38.70 \%, 9.57 \%$, and $56.62 \%$ for corn chips, promasa tortillas, and wheat flour tortillas, respectively. Moreover, the percentage of use was increased, and the percentage of waiting time for each operation was reduced. With this, efficiency was enhanced through the increased production capacity.
\end{abstract}

Keywords: Discrete event simulation, production processes, resource allocation, throughput, process improvement.

\footnotetext{
1 Universidad de La Salle. Departamento de Ingeniería Industrial. Bogotá, Colombia. E-mail: 1pena22@unisalle.edu.co

* Autor de correspondencia: healfelizzola@unisalle.edu.co
} 


\section{INTRODUCCIÓN}

En la actualidad, se requiere ser cada día más competitivos mediante la optimización de los procesos, esto implica mejorar la utilización y asignación de los recursos que intervienen en la producción [1]. Para muchas empresas la competitividad se define de distintas formas, pero en este caso se caracteriza como la capacidad de una empresa para producir sus productos, usando los recursos eficientemente, de tal forma que permita ser más competitivos; ya que al optimizar la capacidad de producción se puede hacer más, empleando los mismos recursos [2].

En las organizaciones es fundamental la toma de decisiones, ya que a partir de estas se generan distintos impactos en los procesos productivos. Por ende, las empresas pequeñas y medianas se basan en técnicas de toma de decisiones fundamentadas en el conocimiento adquirido por la experiencia, sin la posibilidad de verificar la efectividad sus decisiones [3]. Es importante que las empresas pequeñas y medianas incluyan dentro de sus herramientas básicas una que sea útil para la toma de decisiones, que no represente una inversión significativa, y que permita desarrollar experimentos en el proceso de una organización [4]. Una herramienta usada actualmente por muchas compañías es la simulación computacional, la cual se utiliza con el fin de modificar los recursos, procesos, políticas, entre otros, sin necesidad de intervenir y modificar el sistema de producción actual [5].

Existen diversos trabajos que utilizan la simulación de eventos discretos para optimizar los procesos productivos según la necesidad que tiene cada empresa. Rivadeneira [6] simuló el proceso de recepción de materia prima de una empresa de curtiembre, y se desarrollaron dos modelos de simulación: el primero, del funcionamiento actual del proceso, y el segundo del proceso automatizado, con ello se obtendría un incremento en la productividad, principalmente en la reducción de tiempos en cada operación y adicionalmente disminución en los costos de producción. Sánchez y Ceballos [7], realizan el análisis de una empresa dedicada a la confección de ropa exterior masculina, con el fin de incrementar la productividad, para ello se desarrolló un modelo del sistema real con la herramienta Arena ${ }^{\circledR}$, para identificar las falencias en el proceso actual y proponer escenarios de solución orientados a incrementar la productividad de la empresa. Solano, Bravo y Giraldo [8], modelan el sistema de producción de una empresa clasificada en PyME usando simulación de eventos discretos con el fin de experimentar diversas alternativas de mejoramiento que estuvieran orientadas a las prioridades competitivas más relevantes, y se obtuvo como resultado el aumento del indicador de efectividad. Pernía, Ramírez y Márquez [9], simularon la línea de producción de la Empresa Alimentos Pepsico S.A, donde se plantearon distintos escenarios tales como el aumento y la disminución de la demanda del producto, donde los resultados indican que la empresa puede aumentar la capacidad en una de sus operaciones, con el fin de aumentar su rendimiento. Garriz y Domingo [10], modelaron una célula de producción de automóviles, donde analizaron el sistema actual y simularon escenarios basados en productividad, datos y variables, teniendo en cuenta que estos supuestos fueran aptos para implementarlos en la producción actual, y obtener una mejora significativa. Omogbaia y Salonitis [11], utilizan la simulación de eventos discretos para modelar un sistema lean manufacturing y proponen distintos escenarios, con el fin de obtener mejoras que sean aplicables a la producción actual. Sachidanandaa, Erkoyuncua, Steenstraa y Michalskaa [12], simularon una compañía biofarmacéutica, para mejorar los procesos de fabricación y plantear escenarios hipotéticos, y obtener una mejora significativa, con respecto al proceso actual en términos de reducción de tiempo, mejor utilización de recursos, reducción de costes operativos, reducción de cuellos de botella. Mejía y Galofre [13], usaron la simulación como herramienta de apoyo en la toma de decisiones, para el rediseño de plantas de producción en empresas del sector de alimentos, obteniendo una mejora en el tiempo de espera, unidades en el sistema y utilización de recursos. Calvo y Motta [14], analizaron el sistema logístico, en una compañía multinacional perteneciente al sector de alimentos y evaluaron la efectividad del proceso actual mediante la elaboración de un modelo

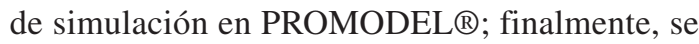
desarrollaron escenarios, con el fin de encontrar mejoras significativas frente a la situación actual en cuanto a costo y tiempo. Guerrero y Henríques [15], modelan las operaciones logísticas que intervienen en la exportación marítima, usando 
simulación para variar los principales parámetros y variables del sistema, con el fin de proponer distintos escenarios orientados a la planeación de despachos; los escenarios propuestos muestran que es posible obtener una reducción de los inventarios y los costos de mantenimiento, y adicionalmente aumentar la tasa de utilización de los recursos. Morales, Hernández y Jiménez [16], modelan un sistema productivo a través de redes de Petri, para estudiar el comportamiento y apoyar la toma de decisiones para mejorar estratégicamente el sistema mediante la integración del paradigma de producción esbelta.

El objetivo de este trabajo se centró en el análisis del proceso productivo de una empresa del sector de alimentos, con el fin de evaluar propuestas de mejora en la capacidad del proceso de producción y por ende la eficiencia, identificando las operaciones que marcaban el ritmo de producción, y a partir de esto proponer distintas estrategias. La empresa tuvo la necesidad de evaluar su estado actual, para verificar sus políticas, y recursos actuales de producción, para optimizar la capacidad y satisfacer el requerimiento de nuevos clientes; se evaluaron distintos escenarios variando políticas de producción y asignación de recursos con el fin de aumentar la eficiencia, esta medición se realiza por medio de indicadores claves de desempeño: Throughput, porcentaje tiempo de espera, porcentaje de utilización de cada operación de trabajo en la empresa. Para simular el proceso actual y los respectivos escenarios se usó el software Tecnomatix Plant Simulation. El aporte principal consiste en mostrar al sector productivo específicamente el alimenticio, la utilidad de la incorporación de la simulación de eventos discretos para la toma de decisiones, integrado a distintas herramientas como ingeniería de métodos, diseño de experimentos y programación de la producción, logrando la optimización de la capacidad de producción.

\section{METODOLOGÍA}

La metodología se desarrolló en cinco (5) fases, con sus respectivas actividades, como se muestra en la Figura 1.

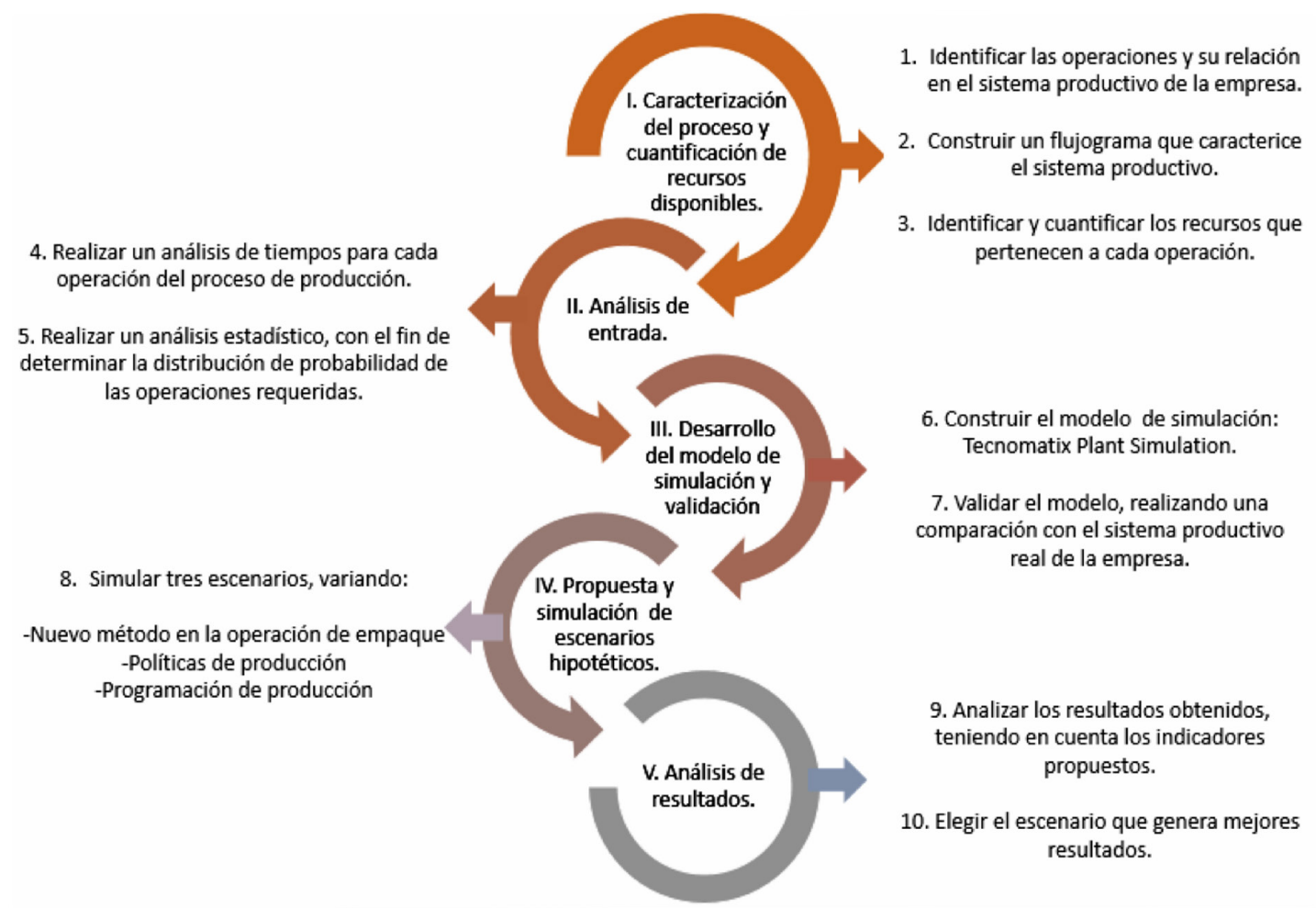

Figura 1. Metodología de desarrollo con sus respectivas fases y actividades. 


\section{Fase I}

Caracterización del proceso y cuantificación de los recursos actuales:

se realizó la identificación de las operaciones que intervienen en el sistema productivo de la empresa y la relación entre las operaciones según el tipo de producto; el sistema se caracteriza por medio de un flujograma y adicionalmente se cuantifican los recursos disponibles para cada operación.

\section{Fase II}

Análisis de entrada:

se realizó un análisis de tiempos para cada operación y por medio de ella, se identificaron operaciones estandarizadas, las cuales tenían tiempo constante y operaciones no estandarizadas a las que se les realizó su respectivo muestreo y análisis estadístico, en el que se aplican distintas pruebas tales como independencia, homogeneidad y bondad de ajuste.

\section{Fase III}

Desarrollo del modelo de simulación y validación: La construcción del modelo, se hizo con la herramienta Tecnomatix Plant Simulation, teniendo en cuenta la información obtenida en la fase I y II. La validación del modelo actual se realiza por medio de una comparación con el estado real del sistema productivo de la empresa con el fin de verificar su validez.

\section{Fase IV}

\section{Propuesta y simulación escenarios:}

En esta fase se plantean tres escenarios y se simulan:

- Nuevo método en la operación de empaque.

- Variación de políticas actuales de producción.

- Reprogramación de la producción actual.

\section{Fase V}

Análisis de resultados:

con los resultados obtenidos, se realizó la comparación entre el escenario actual y los escenarios propuestos, y a partir de esto se seleccionó el escenario que generó mejores resultados, en cuanto a cada indicador.

\section{DESARROLLO DE LA METODOLOGÍA PROPUESTA}

\section{Fase I: Caracterización del proceso y cuantificación de los recursos actuales \\ Proceso productivo}

La empresa cuenta con tres productos: tortillas de maíz, tortillas de promasa, tortillas de harina de trigo, y cada uno de estos tiene un proceso diferente; para caracterizar el proceso de cada producto se construyó un diagrama de operaciones.

En la producción de tortillas de maíz, se siguen las operaciones que se muestran en el Diagrama 1, iniciando su proceso con lavado y finalizando con empacado; el tiempo y las cantidades que se presentan corresponden a un lote de 50 libras de maíz, es decir 62 docenas de tortillas de maíz con un tiempo de ciclo de 312,36 minutos. En la producción de tortillas de promasa, se siguen las operaciones que se muestran en el Diagrama 1, iniciando su proceso con amasado y finalizando con empacado; el tiempo y las cantidades que se presentan corresponden a un lote de 50 libras de promasa, es decir 112 docenas de tortillas de promasa con un tiempo de ciclo de 217 minutos. En la producción de tortillas de harina de trigo, se siguen las operaciones que se muestran en el Diagrama 1, iniciando su proceso con amasado y finalizando con empacado; el tiempo y las cantidades que se presentan corresponden a un lote de 50 libras de harina de trigo, es decir 84 docenas de tortillas de harina de trigo con un tiempo de ciclo de 410 minutos.

La caracterización general del sistema productivo, se representa por medio de un flujograma en el que se fusionan todas las operaciones y se observa de forma más clara el comportamiento del sistema de acuerdo a cada materia prima (ver Figura 2). El proceso inicia con la entrada de la materia prima, si la materia prima es maíz, se da inicio con la operación de lavado, posteriormente de desarrollan las operaciones de cocinado, molido y amasado; de lo contrario, si la materia prima es promasa o harina de trigo, se da inicio con la operación de amasado; después se generan tres opciones según el tipo de producto: maíz, promasa y harina de trigo, si este es maíz debe pasar por la operación tortillar maíz y finalizar en empacado, si esta es promasa debe pasar por tortillar promasa y finalizar en empacado, si este es harina de trigo debe pasar por boleadora, posteriormente de desarrollan las operaciones de tortillar harina de trigo, corte y finalmente en empacado; al terminar los tres productos en empacado se da por terminado el sistema productivo.

Para la cuantificación de recursos actuales, se construyó el Cuadro 1 donde se muestran los procesos, recursos vs. los productos, con el fin de 


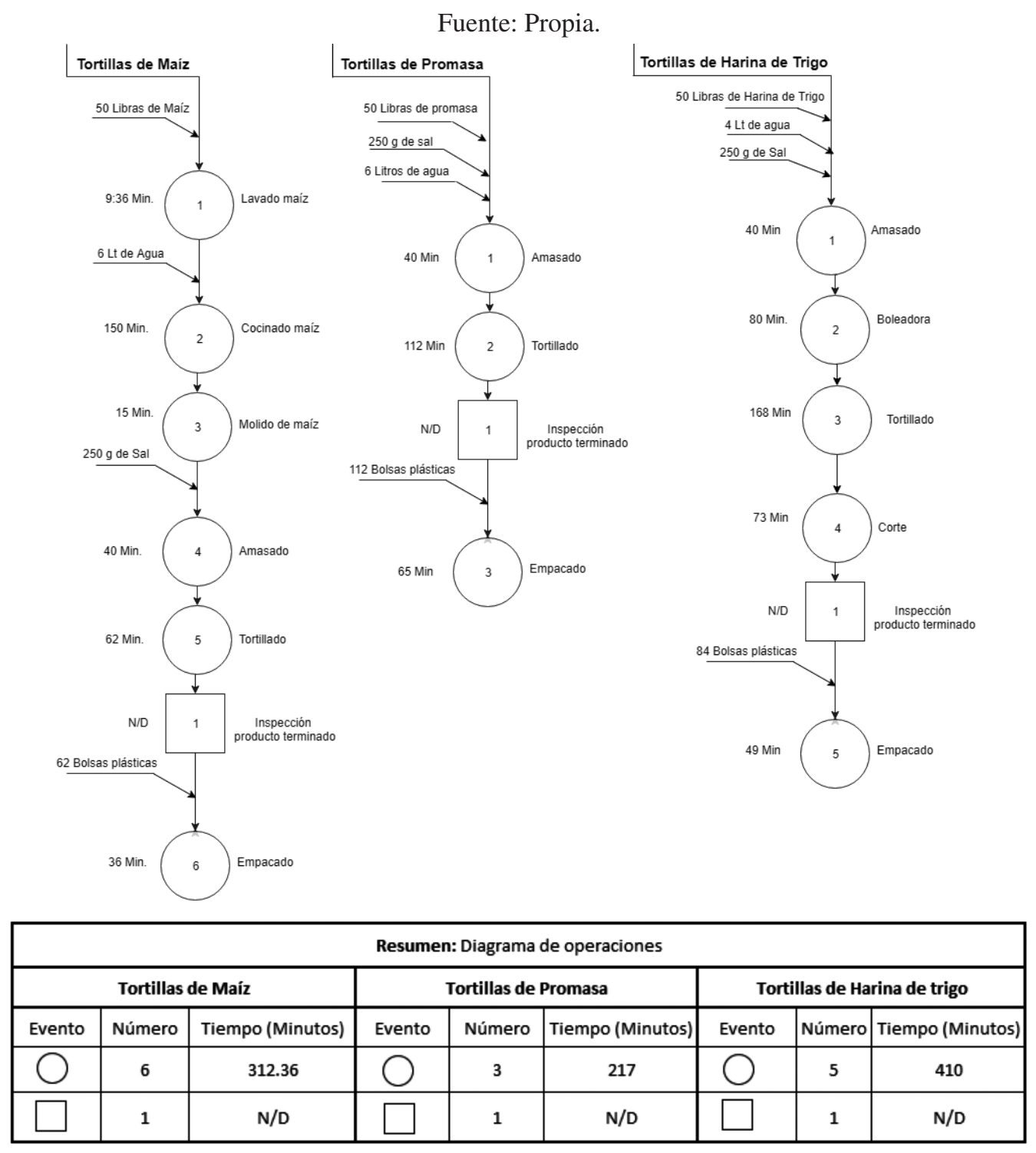

Diagrama 1. Diagrama de operaciones tortillas de maíz, tortillas de promasa y tortillas de harina de trigo.

establecer la relación y la cuantificación de los recursos requeridos por cada producto en cada operación. En las operaciones de amasar y empaque, se comparten los recursos para los tres productos, es decir que la empresa actualmente solo cuenta con una (1) amasadora y una (1) mesa de empaque y se usa en el proceso para cada producto según la disponibilidad que tenga.

\section{Fase II: Análisis de entrada}

En cuanto al análisis de entrada, se realizó la toma de tiempos que se asocia a cada operación del sistema productivo, para ello se tiene en cuenta que procesos están estandarizados y cuales no; al realizar esta identificación se determinó que los procesos estandarizados son: cocinar, moler, amasar, bolear, tortillar maíz, tortillar promasa, tortillar harina de trigo, es decir que estos procesos tienen tiempo constante; las operaciones no estandarizadas son: lavado, corte y empaque, para estas se realizó un muestreo de 30 datos durante una semana, ya que era el tiempo que disponía la empresa para realizar dicho análisis, teniendo en cuenta que la producción diaria es diferente durante la semana, y la empresa 
Fuente: Propia

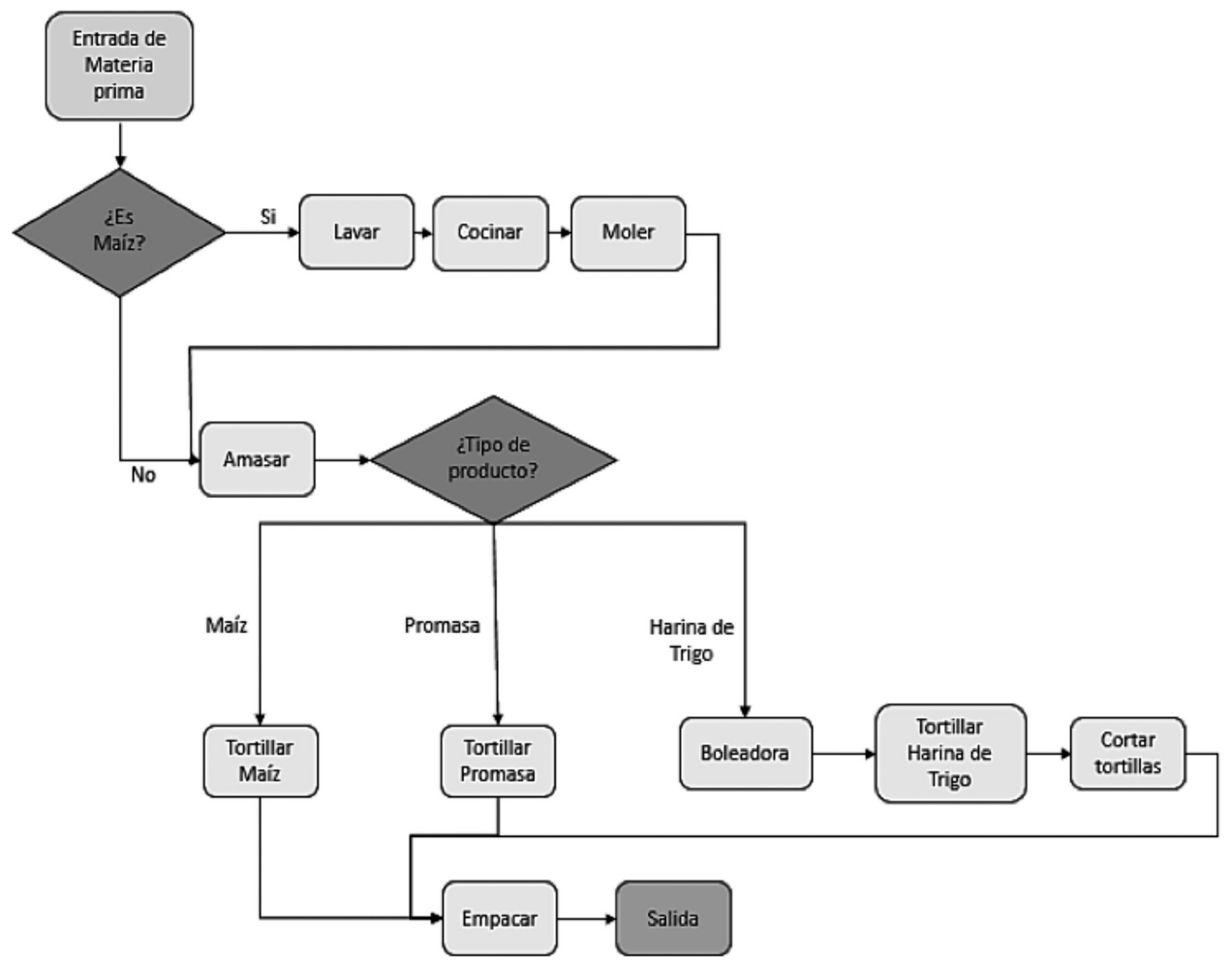

Figura 2. Flujograma, Caracterización sistema productivo.

Cuadro 1. Cuantificación de recursos físicos disponibles en cada proceso por producto.

\begin{tabular}{|l|l|c|c|c|}
\hline \multicolumn{2}{|c|}{ Proceso } & \multicolumn{3}{c|}{ Productos [Recursos utilizados] } \\
\hline Operación & \multicolumn{1}{|c|}{ Recurso } & $\begin{array}{c}\text { Tortillas } \\
\text { de maíz }\end{array}$ & $\begin{array}{c}\text { Tortillas } \\
\text { de promasa }\end{array}$ & $\begin{array}{c}\text { Tortillas de } \\
\text { Harina de trigo }\end{array}$ \\
\hline Lavar & Malla & 1 & No aplica & No aplica \\
\hline Cocinar & Estufa & 2 & No aplica & No aplica \\
\hline Moler & Molino & 1 & No aplica & No aplica \\
\hline Amasar & Amasadora & No aplica & No aplica & 1 \\
\hline Bolear & Boleadora & 1 & No aplica & No aplica \\
\hline Tortillar 1 & Tortilladora de maíz & No aplica & 1 & No aplica \\
\hline Tortillar 2 & Tortilladora de promasa & No aplica & No aplica & 1 \\
\hline Tortillar 3 & Tortilladora de trigo & No aplica & 1 \\
\hline Zona de corte & Mesa de corte & No aplica & No & \multicolumn{4}{|l}{} \\
\hline Zona de empaque & Mesa de empaque & \multicolumn{4}{|c|}{} \\
\hline
\end{tabular}

Fuente: Propia.

labora de lunes a viernes, en un horario comprendido entre 7:00 am a 5:00 pm, con una hora de receso.

En el Cuadro 2, se muestran los resultados obtenidos en la prueba de independencia, homogeneidad y bondad de ajuste; la prueba de independencia, se realizó por medio del método grafico de auto correlación y se evidencio que los datos recolectados en la operación de lavado, corte y empaque, clasifican en el rango de correlación débil, es decir que son 
Cuadro 2. Resumen pruebas, análisis de entrada.

\begin{tabular}{|c|c|c|c|}
\hline Pruebas: & Independencia & Homogeneidad & Bondad de ajuste \\
\hline Lavado & 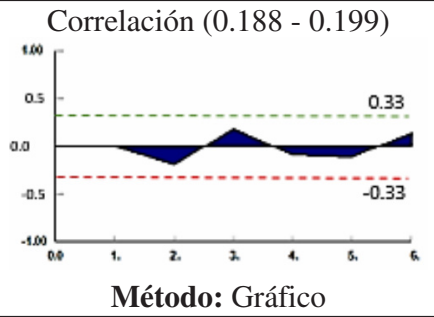 & $\begin{array}{l}\text { Ho: La mediana de datos en cada } \\
\text { día es aproximadamente igual. } \\
\text { Ha: La mediana de datos en } \\
\text { cada día es diferente. } \\
\text { Prueba de Kruskal-Wallis: } \\
\text { No se rechaza la hipótesis nula } \\
\text { Nivel de Significancia: } 0,05 \text {. }\end{array}$ & $\begin{array}{l}\text { Prueba: } \\
\text { Kolmogorov-Smirnov } \\
\text { Valor p: } 0.226 \\
\text { Distribución: } \\
\text { Log normal }(66.1 \%)\end{array}$ \\
\hline Zona de Corte & $\begin{array}{l}\text { Correlación (0.145 - 0.27) } \\
\text { Método: Gráfico }\end{array}$ & $\begin{array}{l}\text { Ho: La mediana de datos en cada } \\
\text { día es aproximadamente igual. } \\
\text { Ha: La mediana de datos en } \\
\text { cada día es diferente. } \\
\text { Prueba de Kruskal-Wallis: } \\
\text { No se rechaza la hipótesis nula } \\
\text { Nivel de Significancia: } 0,05 \text {. }\end{array}$ & $\begin{array}{l}\text { Prueba: } \\
\text { Kolmogorov-Smirnov } \\
\text { Valor p: } 0.219 \\
\text { Distribución: } \\
\text { Log normal }(60.2 \%)\end{array}$ \\
\hline $\begin{array}{l}\text { Zona de } \\
\text { empaque }\end{array}$ & 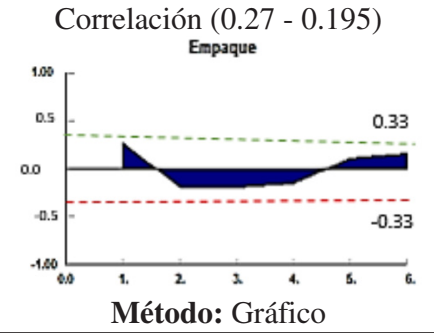 & $\begin{array}{l}\text { Ho: La mediana de datos en cada } \\
\text { día es aproximadamente igual. } \\
\text { Ha: La mediana de datos en } \\
\text { cada día es diferente. } \\
\text { Prueba de Kruskal-Wallis: } \\
\text { No se rechaza la hipótesis nula } \\
\text { Nivel de Significancia: } 0,05 \text {. }\end{array}$ & $\begin{array}{l}\text { Prueba: } \\
\text { Kolmogorov-Smirnov } \\
\text { Valor p: } 0.223 \\
\text { Distribución: } \\
\text { Log normal }(100 \%)\end{array}$ \\
\hline
\end{tabular}

Fuente: IBM SPSS Statistics y ExperFit-Promodel.

independientes; se verifico la homogeneidad de los datos usando la prueba de Kruskal-Wallis; en la prueba de bondad de ajuste, se seleccionó la distribución de probabilidad para cada operación, teniendo en cuenta la precisión y la compatibilidad con el modelo en Tecnomatix Plant Simulation.
En la Tabla 1, se muestran los parámetros usados para el modelo de simulación, se determinó que la distribución de las operaciones: cocinar, moler, amasar, bolear, tortillar 1, tortillar 2 y tortillar 3, es constante por que el tiempo esta parametrizado por la empresa y no tiene variaciones significativas.

Tabla 1. Parámetros de entrada, modelos de simulación.

\begin{tabular}{|l|l|l|l|}
\hline \multicolumn{1}{|c|}{ Operación } & Distribución & Parámetros de la distribución & \multicolumn{1}{|c|}{$\begin{array}{c}\text { Valor del parámetro } \\
\text { Horas : Minutos : Segundos }\end{array}$} \\
\hline Lavar & Lognormal & Mu, Sigma & $09: 37,0: 08.4949$ \\
\hline Cocinar & Constante & Hora : Minutos : Segundo & $02: 30: 00$ \\
\hline Moler & Constante & Hora : Minutos : Segundo & $00: 15: 00$ \\
\hline Amasar & Constante & Hora : Minutos : Segundo & $00: 40: 00$ \\
\hline Bolear & Constante & Hora : Minutos : Segundo & $01: 20: 00$ \\
\hline Tortillar 1 & Constante & Hora : Minutos : Segundo & $00: 01: 00$ \\
\hline Tortillar 2 & Constante & Hora : Minutos : Segundo & $00: 01: 00$ \\
\hline Tortillar 3 & Constante & Hora : Minutos : Segundo & $00: 02: 00$ \\
\hline Zona de corte & Lognormal & $M u$, Sigma & $00: 00: 54,00: 00: 01.3978$ \\
\hline Zona de empaque & Lognormal & $M u$, Sigma & $00: 00: 38,00: 00: 01.6274$ \\
\hline
\end{tabular}


Fase III: Desarrollo del modelo de simulación y validación

\section{Indicadores claves de desempeño}

Para la medición de la eficiencia del proceso se plantearon tres indicadores claves de desempeño:

- Throughput, corresponde al número de unidades terminadas en un periodo de tiempo,

- Porcentaje tiempo de espera en cada operación,

- Porcentaje de utilización en cada operación.

Para la construcción del modelo en Tecnomatix Plant Simulation, se tuvo en cuenta el desarrollo de la fase I: Caracterización del proceso y fase II: Análisis de entrada.

Es importante resaltar que la entrada del sistema es materia prima por lotes de 50 libras cada uno: (maíz, promasa, harina de trigo), y la salida es: tortillas de maíz $=62$ docenas, tortillas de promasa $=112$ docenas, tortillas de harina de trigo $=84$ docenas por cada lote. La producción de la empresa es diferente durante la semana, es decir, que se construyeron dos modelos de simulación variando la programación de la producción, teniendo en cuenta que el esquema del modelo no cambia; el modelo 1 corresponde a la producción de lunes, miércoles y viernes, donde se producen tortillas de maíz y tortillas de promasa y el modelo 2 corresponde a la producción de martes y jueves, donde se producen tortillas de maíz y tortillas de harina de trigo. En la Figura 3 se muestra el esquema que arroja la interfaz del modelo de simulación actual. La simulación se ejecutó durante dos semanas, ya que es el esquema de producción que maneja la empresa.

\section{Análisis estado actual}

En la Tabla 2, se muestran los resultados obtenidos del escenario actual, donde se distinguen los dos modelos, adicionalmente en cada modelo se miden los distintos indicadores; el modelo 1 se simula durante 54 horas equivalentes a dos semanas de producción, el modelo 2 se simula durante 36 horas equivalentes a dos semanas de producción. Las condiciones mencionadas anteriormente, también aplican para el escenario 1 y 2 (Medición de indicadores y tiempo de simulación).

Cuadro 3. Indicadores claves de desempeño.

\begin{tabular}{|c|c|c|c|c|}
\hline $\mathbf{N}^{\mathbf{o}}$ & Nombre del indicador & Unidad de medida & Sentido & Fuente de información \\
\hline 1 & Throughput & Número & Creciente & Tecnomatix Plant Simultion \\
\hline 2 & $\begin{array}{l}\text { Porcentaje tiempo de espera en } \\
\text { cada estación }\end{array}$ & Porcentaje & Decreciente & Tecnomatix Plant Simultion \\
\hline 3 & $\begin{array}{l}\text { Porcentaje de utilización en } \\
\text { cada estación }\end{array}$ & Porcentaje & Creciente & Tecnomatix Plant Simultion \\
\hline
\end{tabular}

Fuente: Tecnomatix Plant Simulation.

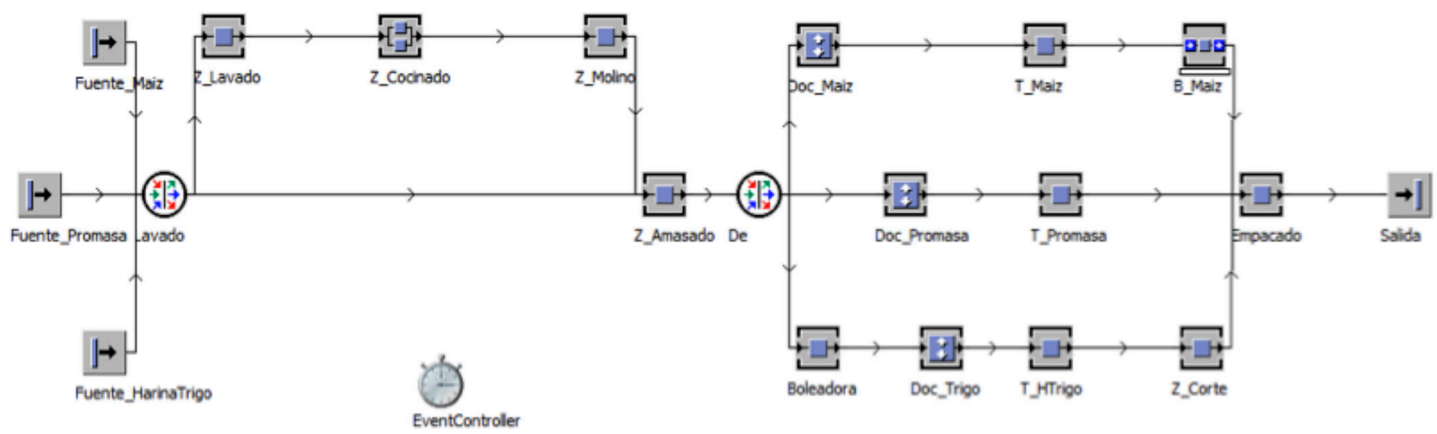

Figura 3. Representación modelo de simulación actual. 
Tabla 2. Resultados indicadores escenario actual.

\begin{tabular}{|l|c|c|c|c|}
\hline \multirow{2}{*}{ Operación } & \multicolumn{2}{|c|}{ Modelo 1 } & \multicolumn{2}{c|}{ Modelo 2 } \\
\cline { 2 - 5 } & $\begin{array}{c}\text { Porcentaje de } \\
\text { utilización }\end{array}$ & $\begin{array}{c}\text { Porcentaje de } \\
\text { tiempo de espera }\end{array}$ & $\begin{array}{c}\text { Porcentaje de } \\
\text { utilización }\end{array}$ & $\begin{array}{c}\text { Porcentaje de tiempo } \\
\text { de espera }\end{array}$ \\
\hline Lavar & $8 \%$ & $0 \%$ & $6,29 \%$ & $0,00 \%$ \\
\hline Cocinar & $97,59 \%$ & $0,30 \%$ & $83,43 \%$ & $0,44 \%$ \\
\hline Moler & $11,11 \%$ & $4,93 \%$ & $7,73 \%$ & $7,39 \%$ \\
\hline Amasar & $61,73 \%$ & $0 \%$ & $44,54 \%$ & $0 \%$ \\
\hline Bolear & No aplica* & No aplica* & $48,24 \%$ & $1,85 \%$ \\
\hline Tortillar 1 & $44,38 \%$ & $55,62 \%$ & $29,17 \%$ & $70,83 \%$ \\
\hline Tortillar 2 & $89,81 \%$ & $1,23 \%$ & No aplica* & No aplica* \\
\hline Tortillar 3 & No aplica* & No aplica* & $94,44 \%$ & $5,56 \%$ \\
\hline Zona de corte & No aplica* & No aplica* & $40,39 \%$ & $59,61 \%$ \\
\hline Zona de empaque & $74,21 \%$ & $25,79 \%$ & $44,28 \%$ & $55,72 \%$ \\
\hline \multirow{2}{*}{ Producto } & \multicolumn{3}{|c|}{ Modelo 1 } & \multicolumn{2}{c|}{ Modelo 2 } \\
\cline { 2 - 5 } & Tortillas de maíz & Tortillas de promasa & Tortillas de maíz & Tortillas harina de trigo \\
\hline Throughput & 1391 & 2909 & 630 & 1019 \\
\hline
\end{tabular}

* Donde se muestra "No aplica", corresponde a las operaciones que no hacen parte de la elaboración del producto.

\section{Validación del modelo}

En la Gráfica 1, se muestra los resultados de las cinco réplicas que se realizaron para verificar la consistencia del modelo, se comprobó su estabilidad, ya que entre cada replica la variación es mínima. La validación del modelo actual, se realiza por medio de una comparación del throughput real y el obtenido en el modelo de simulación, equivalente a dos semanas de producción. En la Tabla 3, se muestra el throughput por cada producto con su respectivo error experimental; se verifica la validez del modelo, teniendo en cuenta que ningún producto tiene un error experimental superior al $10 \%$.

\section{Fase IV: Propuesta y simulación escenarios}

- Escenario 1: Nuevo método en la operación de eтраque.

El método propuesto en la operación de empaque, se muestra en el diagrama bimanual actual y el propuesto (ver Diagrama 2), donde se puede evidenciar que se eliminaron las actividades innecesarias del proceso actual y se obtuvo una

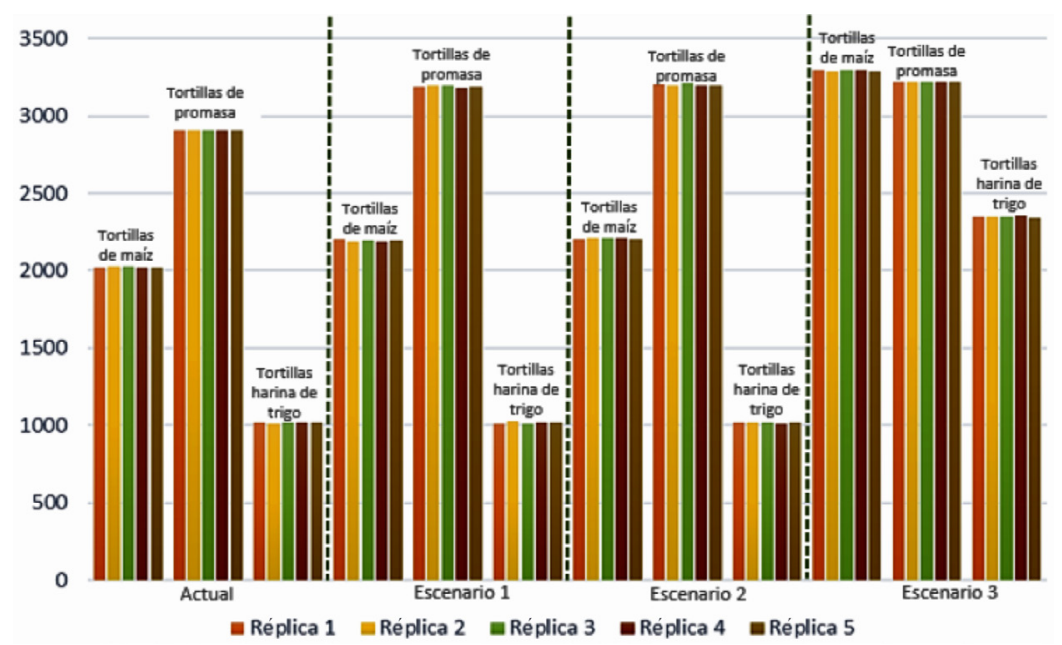

Gráfica 1. Validación estabilidad del modelo. 
Tabla 3. Validación del modelo.

\begin{tabular}{|l|c|c|c|}
\hline & \multicolumn{3}{|c|}{ Throughput: 2 Semanas } \\
\hline \multicolumn{1}{|c|}{ Producto: } & Real & Modelo & Error experimental \\
\hline Tortillas de maíz & 1860 & 2021 & $8,66 \%$ \\
\hline Tortillas de promasa & 2688 & 2909 & $8,22 \%$ \\
\hline Tortillas harina de trigo & 1008 & 1019 & $1,09 \%$ \\
\hline
\end{tabular}

Fuente: Propia.

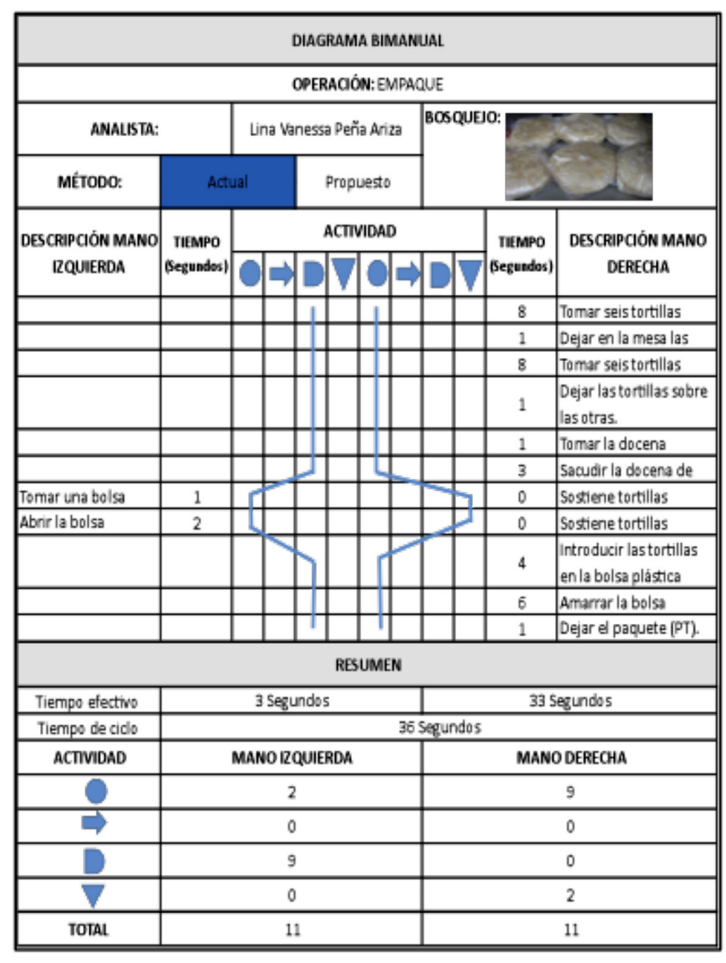

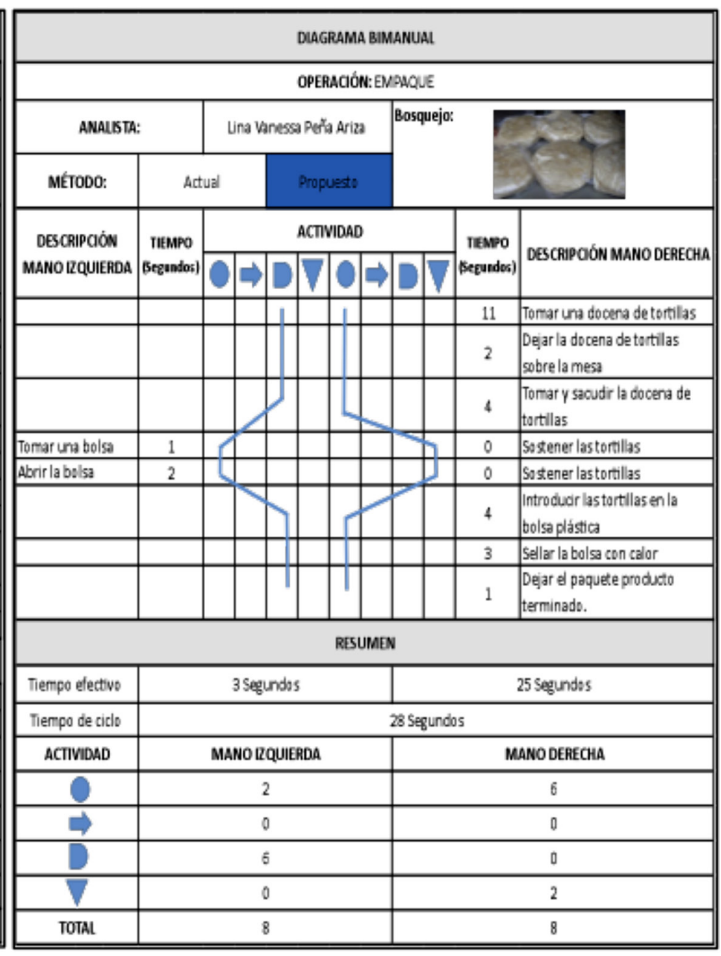

Diagrama 2. Diagrama bimanual: Actual y propuesto, operación de empaque.

reducción de 19,44\% en el tiempo de la operación de empaque. Al método propuesto se le realizó el respectivo muestreo de 30 datos durante una semana y el análisis estadístico correspondiente, prueba de independencia, homogeneidad y bondad de ajuste.

En el Cuadro 4, se muestran los resultados obtenidos en cada una de las pruebas; la prueba de independencia, se realizó por medio del método grafico de auto correlación y se evidencio que la operación de empaque, clasifica en el rango de correlación moderada, es decir que es independiente, adicionalmente se verifico la homogeneidad de los datos usando la prueba de Kruskal-Wallis; en la prueba de bondad de ajuste Kolmogorov-Smirnov, se obtuvo que la distribución de probabilidad de los datos es Log normal.

En la Tabla 4, se muestran los resultados obtenidos en el escenario 1, donde se distinguen los dos modelos.

\section{- Escenario 2: Modificación de políticas de producción} Al verificar las políticas de producción actuales, se evidenció que la empresa realiza la operación de empaque por lotes de producción, es decir, cuando el lote de cada producto estuviera terminado y apilado 
Cuadro 4. Análisis de entrada, método propuesto de empaque.

\begin{tabular}{|c|c|c|c|}
\hline Pruebas: & Independencia & Homogeneidad & Bondad de ajuste \\
\hline $\begin{array}{l}\text { Zona de } \\
\text { empaque }\end{array}$ & Correlación (0.196-0.513) & $\begin{array}{l}\text { Ho: La mediana de datos en cada día } \\
\text { es aproximadamente igual. } \\
\text { Ha: La mediana de datos en cada día } \\
\text { es diferente. } \\
\text { Prueba de Kruskal-Wallis: } \\
\text { No se rechaza la hipótesis nula } \\
\text { Nivel de Significancia: } 0,05\end{array}$ & $\begin{array}{l}\text { Prueba: } \\
\text { Kolmogorov-Smirnov } \\
\text { Valor p: } 0.209 \\
\text { Distribución: } \\
\text { Log normal }(100 \%)\end{array}$ \\
\hline
\end{tabular}

Fuente: IBM SPSS Statistics y ExperFit-Promodel.

Tabla 4. Resultados indicadores escenario 1.

\begin{tabular}{|c|c|c|c|c|}
\hline \multirow[b]{2}{*}{ Operación } & \multicolumn{2}{|c|}{ Modelo 1} & \multicolumn{2}{|c|}{ Modelo 2} \\
\hline & $\begin{array}{c}\text { Porcentaje de } \\
\text { utilización }\end{array}$ & $\begin{array}{l}\text { Porcentaje de } \\
\text { tiempo de espera }\end{array}$ & $\begin{array}{c}\text { Porcentaje de } \\
\text { utilización }\end{array}$ & $\begin{array}{l}\text { Porcentaje de } \\
\text { tiempo de espera }\end{array}$ \\
\hline Lavar & $8,88 \%$ & $0 \%$ & $6,29 \%$ & $0,00 \%$ \\
\hline Cocinar & $97,59 \%$ & $0,30 \%$ & $83,43 \%$ & $0,44 \%$ \\
\hline Moler & $12,50 \%$ & $4,92 \%$ & $7,73 \%$ & $7,39 \%$ \\
\hline Amasar & $67,43 \%$ & $0 \%$ & $44,54 \%$ & $0 \%$ \\
\hline Bolear & No aplica & No aplica & $48,24 \%$ & $1,85 \%$ \\
\hline Tortillar 1 & $48,61 \%$ & $51,39 \%$ & $29,17 \%$ & $70,83 \%$ \\
\hline Tortillar 2 & $98,39 \%$ & $1,23 \%$ & No aplica & No aplica \\
\hline Tortillar 3 & No aplica & No aplica & $94,44 \%$ & $5,56 \%$ \\
\hline Zona de corte & No aplica & No aplica & $40,39 \%$ & $59,61 \%$ \\
\hline Zona de empaque & $71,82 \%$ & $28,19 \%$ & $36,90 \%$ & $63,10 \%$ \\
\hline \multirow[b]{2}{*}{ Producto } & \multicolumn{2}{|c|}{ Modelo 1} & \multicolumn{2}{|c|}{ Modelo 2} \\
\hline & $\begin{array}{l}\text { Tortillas } \\
\text { de maíz }\end{array}$ & $\begin{array}{c}\text { Tortillas } \\
\text { de promasa }\end{array}$ & $\begin{array}{l}\text { Tortillas } \\
\text { de maíz }\end{array}$ & $\begin{array}{c}\text { Tortillas harina } \\
\text { de trigo }\end{array}$ \\
\hline Throughput & 1564 & 3187 & 630 & 1019 \\
\hline
\end{tabular}

antes de dicha operación, por ende, se aumentaba el tiempo de espera en las operaciones que antecedían la operación de empaque como se muestra en la Tabla 2; a partir de esto se eliminó dicha restricción y el empaque se realizó cuando la docena de cualquier producto estuviese terminada; adicionalmente el modelo se ejecutó en este escenario, teniendo en cuenta el tiempo del método propuesto en el escenario 1. En la Tabla 5, se muestran los resultados obtenidos en el escenario 2 .

\section{-Escenario 3: Reprogramación de la producción}

En este escenario se propuso realizar la reprogramación de la producción actual de la empresa, porque al verificar los porcentajes de utilización del estado actual en las operaciones (Tabla 2), se evidenció que los niveles de utilización son en su mayoría inferiores al $50 \%$, por ende, se propuso la fabricación de los tres productos el mismo día, con el fin de aumentar los niveles de utilización. El modelo se simula durante 90 horas equivalentes a dos semanas de producción, teniendo en cuenta el tiempo del método propuesto en el escenario 1 y la política de producción propuesta en el escenario 2. En la Tabla 6, se muestran los resultados obtenidos en el escenario 3, donde se evalúa un solo modelo. 
Tabla 5. Resultados indicadores escenario 2.

\begin{tabular}{|c|c|c|c|c|}
\hline \multirow{2}{*}{ Operación } & \multicolumn{2}{|c|}{ Modelo 1} & \multicolumn{2}{|c|}{ Modelo 2} \\
\hline & $\begin{array}{c}\text { Porcentaje de } \\
\text { utilización }\end{array}$ & $\begin{array}{c}\text { Porcentaje de } \\
\text { tiempo de espera }\end{array}$ & $\begin{array}{c}\text { Porcentaje de } \\
\text { utilización }\end{array}$ & $\begin{array}{l}\text { Porcentaje de } \\
\text { tiempo de espera }\end{array}$ \\
\hline Lavar & $8,67 \%$ & $0 \%$ & $6,29 \%$ & $0,00 \%$ \\
\hline Cocinar & $98,19 \%$ & $0,22 \%$ & $83,43 \%$ & $0,44 \%$ \\
\hline Moler & $12,50 \%$ & $3,69 \%$ & $7,73 \%$ & $7,39 \%$ \\
\hline Amasar & $68,52 \%$ & $0 \%$ & $44,54 \%$ & $0 \%$ \\
\hline Bolear & No aplica & No aplica & $48,24 \%$ & $1,85 \%$ \\
\hline Tortillar 1 & $50,96 \%$ & $48,65 \%$ & $29,17 \%$ & $70,65 \%$ \\
\hline Tortillar 2 & $99,07 \%$ & $0,93 \%$ & No aplica & No aplica \\
\hline Tortillar 3 & No aplica & No aplica & $94,44 \%$ & $5,56 \%$ \\
\hline Zona de corte & No aplica & No aplica & $40,39 \%$ & $59,38 \%$ \\
\hline Zona de empaque & $73,45 \%$ & $27,50 \%$ & $37,38 \%$ & $62,62 \%$ \\
\hline \multirow[b]{2}{*}{ Producto } & \multicolumn{2}{|c|}{ Modelo 1} & \multicolumn{2}{|c|}{ Modelo 2} \\
\hline & $\begin{array}{l}\text { Tortillas } \\
\text { de maíz }\end{array}$ & $\begin{array}{c}\text { Tortillas } \\
\text { de promasa }\end{array}$ & $\begin{array}{l}\text { Tortillas } \\
\text { de maíz }\end{array}$ & $\begin{array}{l}\text { Tortillas harina } \\
\text { de trigo }\end{array}$ \\
\hline Throughput & 1575 & 3199 & 630 & 1019 \\
\hline
\end{tabular}

Tabla 6. Resultados indicadores escenario 3.

\begin{tabular}{|c|c|c|}
\hline \multirow{2}{*}{ Operación } & \multicolumn{2}{|c|}{ Indicadores } \\
\hline & Porcentaje de utilización & Porcentaje de tiempo de espera \\
\hline Lavar & $10,14 \%$ & $0 \%$ \\
\hline Cocinar & $99,82 \%$ & $0,18 \%$ \\
\hline Moler & $15,00 \%$ & $2,95 \%$ \\
\hline Amasar & $82,18 \%$ & $0 \%$ \\
\hline Bolear & $41,48 \%$ & $55,94 \%$ \\
\hline Tortillar 1 & $61,07 \%$ & $5,94 \%$ \\
\hline Tortillar 2 & $59,59 \%$ & $8,14 \%$ \\
\hline Tortillar 3 & 87,07 & 12,93 \\
\hline Zona de corte & 37,24 & 43,75 \\
\hline Zona de empaque & $96,33 \%$ & $3,67 \%$ \\
\hline \multicolumn{3}{|c|}{ Throughput } \\
\hline Tortillas de maíz & Tortillas de promasa & Tortillas harina de trigo \\
\hline 3297 & 3217 & 2349 \\
\hline
\end{tabular}

\section{Fase V: Análisis resultados}

\section{- Throughput}

En la Gráfica 2, se muestra el comportamiento del Throughput en cada uno de los escenarios. Al comparar el escenario actual con respecto al escenario 1 y 2 se puede observar que existe una tendencia con pocas variaciones en los tres productos, pero en el escenario 3 , se aumenta el throughput significativamente en la producción de tortillas de maíz y harina de trigo. El objetivo de este indicador es incrementar con respecto al estado actual, por tanto, se puede inferir que el escenario seleccionado es el 3, en este indicador; el incremento con respecto al escenario actual por producto fue $38,70 \%, 9,57 \%, 56,62 \%$ de tortillas de maíz, tortillas de promasa y tortillas de harina de trigo respectivamente. 
Gráfica 2. Comportamiento Throughput, en cada escenario.

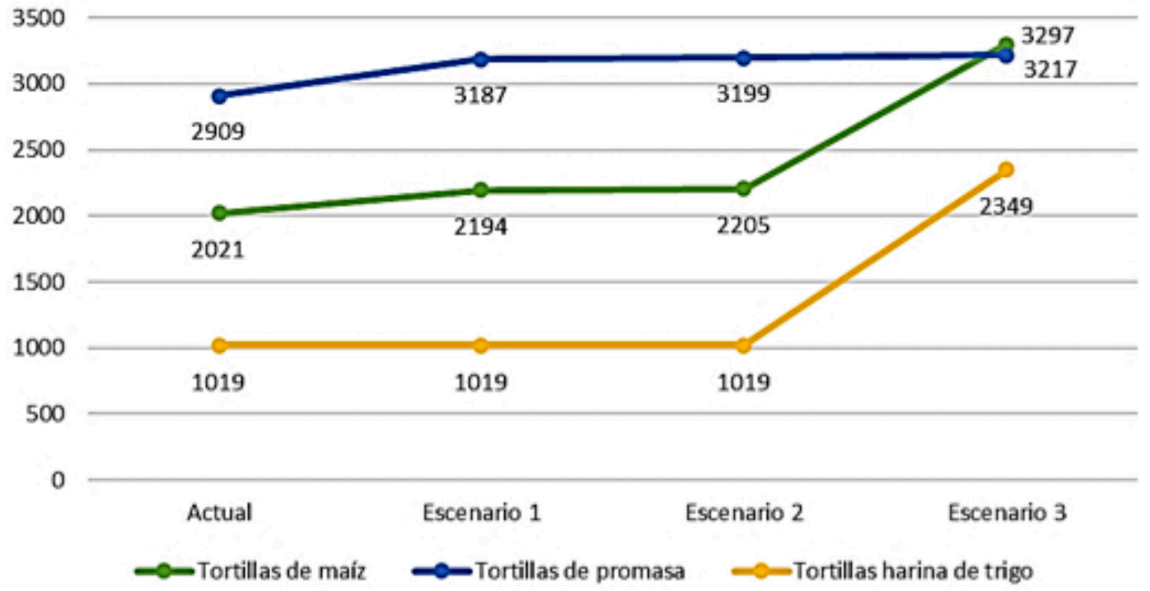

Adicionalmente, se realizó un diseño de experimentos completamente aleatorizado, analizando el throughput como variable de respuesta, para cada producto.

En la Tabla 7, se muestran los resultados obtenidos en el ANOVA por cada producto, donde se rechaza la hipótesis nula y se infiere que cada escenario propuesto tiene una influencia significativa sobre el throughput de cada producto. Adicionalmente, se realizó una comparación de los escenarios usando el método LSD de Fisher (Ver Tabla 8), se concluye que el escenario 3, tiene una influencia significativa frente al escenario actual, en el throughput de cada producto, por ende, es el escenario seleccionado frente a este indicador.

\section{- Porcentaje tiempo de espera}

En la Gráfica 3, se omite del análisis la operación de lavado y amasado, ya que en estas la espera corresponde al $0 \%$. Al comparar el escenario actual con respecto al escenario 1,2 se evidencia que existe una variación mínima en todas las operaciones, adicionalmente existe un pico superior al 50\% en la operación Tortillar 1 y corte. En el escenario 3, se reducen significativamente los porcentajes de tiempo en espera, con respecto al actual en $51,4 \%, 52,1 \%, 90,6 \%, 26,6 \%, 91,0 \%$ para las operaciones cocinar, moler, tortillar 1 , corte y empaque respectivamente, pero en este se observa un efecto inverso al objetivo de este indicador, y se aumenta en $84,9 \%, 96,7 \%$ y $57 \%$ en la operación tortillar 2, bolear y tortillar 3, respectivamente. El objetivo de este indicador es disminuir con respecto al estado actual, por tanto, se puede determinar que el escenario seleccionado es el 3 , para este indicador.

\section{- Porcentaje de utilización}

En la Gráfica 4, se muestra el comportamiento del porcentaje de utilización en cada uno de los

Tabla 7. Resumen del ANOVA para Throughput de cada producto.

\begin{tabular}{|c|c|c|c|}
\hline \multicolumn{4}{|c|}{$\begin{array}{l}\text { Hipótesis nula (Ho): Todas las medias son iguales } \\
\text { Hipótesis alterna (Ha): Por lo menos una media es diferente } \\
\text { Nivel de significancia } \alpha=0,05\end{array}$} \\
\hline \multicolumn{4}{|c|}{ ANOVA } \\
\hline Producto: & Maíz & Promasa & Harina de trigo \\
\hline Cuadrado medio: Tratamientos & 1694552,18 & 110820,85 & 2215339,92 \\
\hline Cuadrado medio: Error & 12,58 & 15,95 & 15,30 \\
\hline Valor F & 134755,64 & 6948,02 & 144793,46 \\
\hline Valor P & 2,00917E-35 & $3,99795 \mathrm{E}-25$ & $1,13086 \mathrm{E}-35$ \\
\hline Resultado: & Se rechaza la Ho & Se rechaza la Ho & Se rechaza la Ho \\
\hline
\end{tabular}


Tabla 8. Resultados de comparación múltiple, método LSD Fisher.

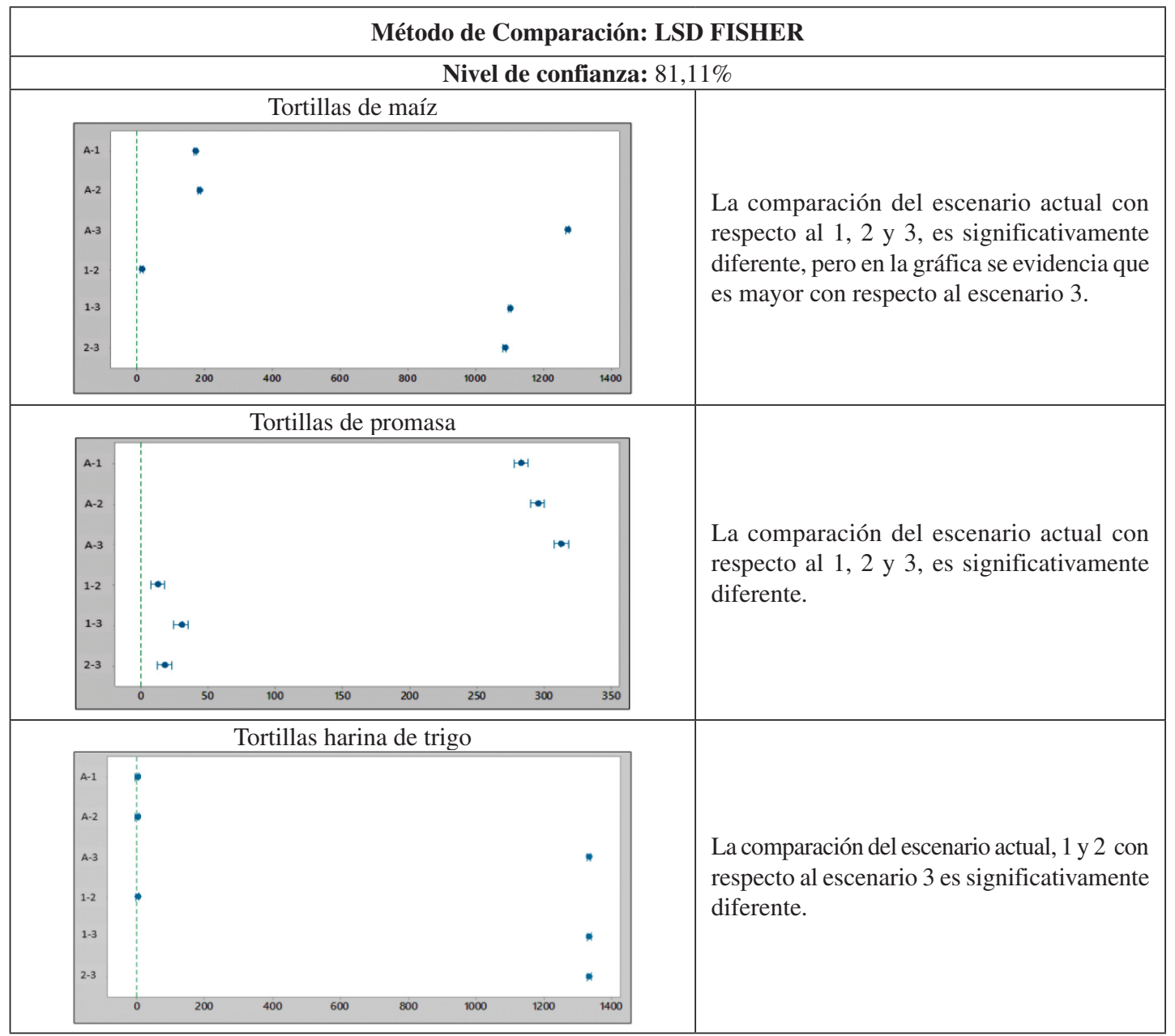

Fuente: Minitab.

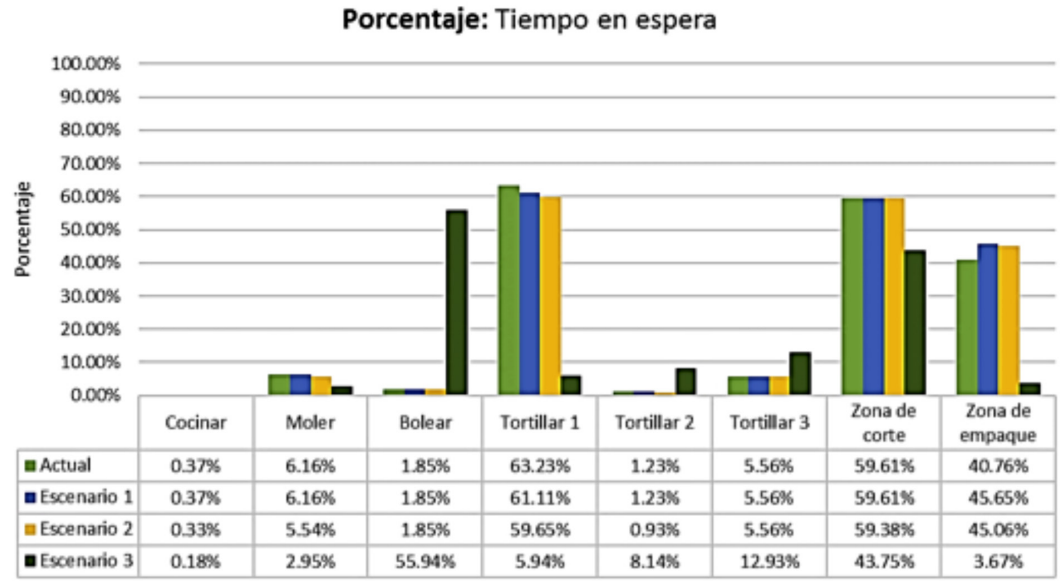

Gráfica 3. Comportamiento porcentaje tiempo de espera en cada operación en los distintos escenarios. 


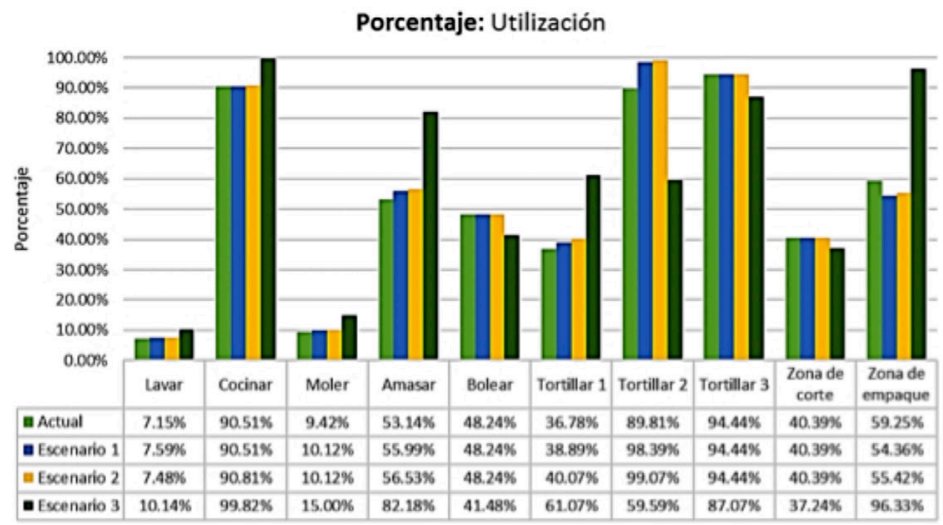

Gráfica 4. Comportamiento porcentaje de utilización en cada operación en los distintos escenarios.

escenarios. Al comparar el escenario actual con respecto al escenario 1 y 2 , se evidencia que existe una variación mínima en todas las operaciones, adicionalmente las operaciones de lavado y molido tienen el porcentaje de utilización inferior al $10 \%$, y la operación de tortillado 1 se mantiene inferior al $40 \%$. En el escenario 3, se evidencia un aumento en el porcentaje de utilización con respecto al escenario actual en la operación de lavado, molido, tortillado 1, empaque, amasado, de 29,54\%, 37,2\%, $39,78 \%, 38,5 \%$ y $35,3 \%$, respectivamente. Pero en este se observa un efecto inverso al objetivo de este indicador, y se reduce el porcentaje de utilización en la operación de tortillar 2, bolear, tortillar 3 y corte en $33,6 \%, 14 \%, 7,8 \%$ y $7,8 \%$, respectivamente. $\mathrm{El}$ objetivo de este indicador es incrementar con respecto al estado actual, por tanto, se determina que el escenario seleccionado es el 3 , en este indicador.

Con los resultados obtenidos en cada indicador, se concluye que el escenario que genera mejores resultados es el 3 , ya que se evidencian variaciones significativas en las operaciones críticas de cada producto.

\section{CONCLUSIONES}

La simulación del escenario actual permitió evidenciar ineficiencia, en la política de producción de la operación de empaque, y en el método actual de dicha operación; adicionalmente la programación de producción actual generaba porcentajes de utilización inferiores al $50 \%$ en el $60 \%$ de las operaciones; y a partir de estos indicadores se plantearon los escenarios. Se simularon tres escenarios de solución orientados a medir tres indicadores de desempeño: Throughput, Porcentaje tiempo de espera, Porcentaje de utilización de cada operación.

Con el análisis y evaluación de resultados, se eligió el escenario 3 para cada uno de los indicadores, ya que se cumplía el objetivo satisfactoriamente; con respecto al Throughput, se generó un aumento por producto de $38,70 \%, 9,57 \%$ y $56,62 \%$ de tortillas de maíz, tortillas de promasa y tortillas de harina de trigo respectivamente, teniendo como base el escenario actual; adicionalmente, se generó una reducción con respecto al porcentaje tiempo de espera del escenario actual en cada operación de 90,60\%, 26,61\%, 90,99\% en la operación de tortillar 1 en las tortillas de maíz, en la operación de corte en las tortillas de harina de trigo y en la operación de empaque para todos los productos respectivamente; finalmente se aumentó el porcentaje de utilización en la operación de lavar, moler, tortillar 1 de $29,54 \%, 37,2 \%, 39,78 \%$, y el $85 \%$ de las otras operaciones se mantienen por encima del $58 \%$. Esto reflejó una utilización óptima de la capacidad, lo cual implica mayores beneficios económicos relacionados con la rentabilidad de la empresa.

Finalmente, se resalta la importancia del uso de la simulación de eventos discretos y del software, ya que en este se incluyeron parámetros claves que influyeron en el éxito de los resultados obtenidos, y no fue necesario hacer pausas en la producción real, para realizar distintos experimentos. Adicionalmente, los escenarios propuestos se pueden implementar en la producción real, y con esto se podría optimizar la capacidad actual de la empresa. 
Como trabajo futuro, se propone evaluar un nuevo escenario, en el que se tenga en cuenta la distribución de planta actual, y validar si la distancia y el tiempo entre las operaciones, tienen una influencia significativa en cuento al resultado de los indicadores, y finalmente evaluar un diseño de distribución de planta, teniendo en cuenta todo lo que esto implica.

\section{REFERENCIAS}

[1] C.S. Navarro Mancilla y I.R. Paz Cisneros. "Optimización en la capacidad de producción de un horno de curvado continuo, para aumentar la productividad en la empresa agp Perú SAC". Universidad privada del Norte. 2016.

[2] N. Labarca y U. Zulia. "Consideraciones teóricas de la competitividad empresarial". Omnia. Vol. 13 N 2. 2007.

[3] G. Gereffi. "Intertiol trade and industrial upgrading in the apparel commodity chain". J. Int. Econ. Vol. 48 Nº 1. 1999.

[4] N. Gilbert. "Computational social science: Agent-based social simulation". Comput. Soc. Sci. Agent-based Soc. Simul., pp. 115-134. 2007. D. Mourtzis and M. Doukas and D. Bernidaki. "Simulation in Manufacturing: Review and Challenges". Procedia CIRP. Vol. 25, pp. 213-229. ISSN: 2212-8271. 2014.

[6] C.S. Rivadeneira Ortiz. "Propuesta de un modelo de simulación en el laboratorio de ingeniería de producción industrial de la Universidad de las Américas, para mejorar la productividad en procesos industriales". 2017.

[7] P.A. Sánchez, F. Ceballos y G. Sánchez Torres. "Análisis del proceso productivo de una empresa de confecciones: modelación y simulación". Ciencia e Ingeniería Neogradi. Vol. $25 \mathrm{~N}^{\circ}$ 2, pp. 137-150. DOI: http://dx.doi. org/10.18359/rcin.1436. 2015.

[8] M. Solano, J. Bravo y J. Giraldo. "Metodología de mejoramiento en el desempeño de sistemas de producción. Aplicaciones en Pymes de la confección". Ingeniería y Competitividad. Vol. $14 \mathrm{~N}^{\mathrm{o}}$ 2, pp. 37-52. 2012.
[9] Y. Pernía, V. Ramírez y R. Márquez. "Un modelo de simulación del proceso de producción de la Línea DTC-2000 de Alimentos Pepsico". Revista Ciencia e Ingeniería. Edición Especial: "Jornada de Modelado y Simulación”, pp. 9-20. 2017.

[10] C. Garriz and R. Domingo. "Simulation, through discrete events, of industrial processes in productive environments". Manufacturing Engineering Society International Conference 2017. MESIC 2017. 28-30 June 2017. Vigo (Pontevedra), Spain. 2017.

[11] O. Omogbaia and K. Salonitis. "Manufacturing system lean improvement design using discrete event simulation". 49th CIRP Conference on Manufacturing Systems (CIRP-CMS 2016). 2016.

[12] M. Sachidanandaa, J. Erkoyuncua, D. Steenstraa and S. Michalskaa. "Discrete Event Simulation Modelling for Dynamic Decision Making in Biopharmaceutical Manufacturing". The Second CIRP Conference on Biomanufacturing. 2016.

[13] H. Mejía y M. Galofre. "Aplicación de software de simulación como herramienta en el rediseño de plantas de producción en empresas del sector de alimentos". Universidad Autónoma del Caribe (UAC): Repositorio Digital. 2008.

[14] J. Calvo García y C. Motta Parra. "Simulación de un evento discreto aplicada a una empresa multinacional XYZ en el sector de alimentos". Universidad ICESI, Colombia: Biblioteca Digital. 2011.

[15] M.A. Guerrero Hernández y A.F. Henriques Librantz. "Simulación de eventos discretos de la cadena logística de exportación de commodities". Ingeniare. Revista chile de ingeniería. Vol. $22 \mathrm{~N}^{\circ}$ 2. 2014.

[16] A. Morales, J. Rojas, L.H. Hernández, A. Morales y M. Jiménez. "Modelo de un sistema de producción esbelto con redes de Petri para apoyar la toma de decisiones". Ingeniare. Revista chile de ingeniería. Vol. 23 $\mathrm{N}^{\circ}$ 2, pp. 182-195. 2015. 